\title{
Decreased expression of DNA repair proteins Ku70 and Mre11 is associated with aging and may contribute to the cellular senescence
}

\author{
Yeun-Jin $\mathrm{Ju}^{1 *}$, Kee-Ho Lee ${ }^{1 *}$, \\ Jeong-Eun Park ${ }^{1}$, Yong-Su Yi ${ }^{1}$, \\ Mi-Yong Yun ${ }^{1}$, Yong-Ho Ham ${ }^{1}$, \\ Tae-Jin Kim², Hyun Mi Choi ${ }^{2}$, \\ Gwi Jung Han ${ }^{3}$, Jong-Hoon Lee ${ }^{4}$, \\ Juneyoung Lee ${ }^{5}$, Jong Seol Han ${ }^{6}$, \\ Kyung-Mi Lee ${ }^{1,7}$ and Gil-Hong Park ${ }^{1,7}$ \\ ${ }^{1}$ Laboratory of Molecular Oncology \\ Korea Institute of Radiological and Medical Sciences \\ Seoul 139-706, Korea \\ ${ }^{2}$ Department of Biochemistry and \\ Division of Brain Korea 21 Program for Biomedical Science \\ College of Medicine, Korea University \\ Seoul 136-705, Korea \\ ${ }^{3}$ National Rural Resources Development Institute \\ Suwon 441-853, Korea \\ ${ }^{4}$ Department of Plastic and Reconstructive Surgery \\ Eulji General Hospital, Eulji University \\ Seoul 139-231, Korea \\ ${ }^{5}$ Department of Preventive Medicine \\ College of Medicine, Korea University \\ Seoul 136-705, Korea \\ ${ }^{6}$ Department of Obstetrics and Gynecology \\ Kangbuk Samsung Hospital \\ School of Medicine, Sungkyunkwan University \\ Seoul 110-746, Korea \\ ${ }^{7}$ Corresponding authors: Tel, 82-2-920-6182; \\ Fax, 82-2-928-4853; E-mail, ghpark@korea.ac.kr, \\ kyunglee@korea.ac.kr \\ *These authors contributed equally to this work.
}

Accepted 9 November 2006

Abbreviations: DSB, double strand break; DNA-PK, DNA-dependent protein kinase; TERC, telomerase RNA component; TERT, telomerase reverse transcriptase; TRF1, telomeric-repeat binding factor 1

\begin{abstract}
The gradual loss of telomeric DNA can contribute to replicative senescence and thus, having longer telomeric DNA is generally considered to provide a longer lifespan. Maintenance and stabilization of telomeric DNA is assisted by binding of multiple
\end{abstract}

DNA-binding proteins, including those involved in double strand break (DSB) repair. We reasoned that declining DSB repair capacity and increased telomere shortening in aged individuals may be associated with decreased expression of DSB repair proteins capable of telomere binding. Our data presented here show that among the DSB repair proteins tested, only the expression of Ku70 and Mre11 showed statistically significant age-dependent changes in human lymphocytes. Furthermore, we found that expressions of Ku70 and Mre11 are statistically correlated, which indicate that the function of Ku70 and Mre11 may be related. All the other DSB repair proteins tested, Sir2, TRF1 and Ku80, did not show any significant differences upon aging. In line with these data, people who live in the regional community (longevity group), which was found to have statistically longer average life span than the rest area, shows higher level of Ku70 expression than those living in the neighboring control community. Taken together, our data show, for the first time, that Ku70 and Mre11 may represent new biomarkers for aging and further suggest that maintenance of higher expression of Ku70 and Mre11 may be responsible for keeping longer life span observed in the longevity group.

Keywords: aging; Ku70; longevity; Mre11A protein; telomere binding proteins; telomere

\section{Introduction}

Telomeres are tandem repeats of the sequence $T_{2} \mathrm{AG}_{3}$ located at the distal end of eukaryotic chromosomes that can function in stabilizing chromosomal end integrity (Prowse and Greider, 1995). Telomeres in somatic cells shorten with age since DNA polymerase is unable to replicate completely the $3^{\prime}$ end of linear DNA. In culture, cells reach a replicative senescence after a certain number of cell divisions (Harley et al., 1990; Harley, 1991), which is thought to be attributed to telomere shortening with each cell replication cycle (Vaziri et al., 1994). In vivo studies in tissues of a variety of eukaryotes have shown a gradual decrease in telomere length related to organism's age (Harley et al., 1990), thus providing a pos- 
sibility that telomere length can be used as a tool to estimate biological age in humans (Haussmann et al., 2003; Hastings et al., 2004; Jeon et al., 2005) . In addition, recent studies revealed that worms genetically engineered to have longer telomeric DNA showed longer lifespan than the corresponding wild type worms (Benard et al., 2001), highlighting the tight control of the lifespan by telomere length. These data suggest that telomere length can serve as a biomarker of a cell biological age.

In contrast to somatic cells, telomere length of germ cells and stem cells do not shorten over time (Bekaert et al., 2004). This can be possible since their telomere length is maintained by the activity of telomerase, an enzyme which adds the necessary telomeric DNA $\left(\mathrm{T}_{2} \mathrm{AG}_{3}\right)$ onto the 3 ' ends of the telomeres (Nilsson et al., 1994). The telomerase holoenzyme consists of a telomerase RNA component (TERC) that serves as a template for the addition of repeats, and a protein component, telomerase reverse transcriptase (TERT). Low or absent expression of telomerase in human somatic tissues is due to the strict regulation of TERT gene transcription, whereas $T E R C$ gene is widely expressed. Thus, up-regulation of TERT expression in somatic cells will be essential for preventing telomere shortening and replicative senescence. Indeed, ectopic expression of human TERT gene was able to rescue senescence of normal human endothelial cells in culture and confer a high proliferative index similar to that of young cells (Haendeler et al., 2004).

Recent studies have revealed that stabilization of chromosome ends by telomeric DNA sequence is assisted by binding of multiple DNA-binding proteins (Wright et al., 2005). These proteins bind to telomeric DNA either directly or indirectly via binding to other proteins bound to telomeric DNA, and form a protective nucleoprotein structure that serves to "cap" the end of the chromosome (Karlseder et al., 2002). The human telomere-repeat binding factor 2 (TRF2) protects chromosome ends from fusion (van Steensel et al., 1998) and was the first telomereassociated protein involved in the maintenance of the correct terminal DNA structure necessary for proper telomere function. Other proteins known to function as DNA repair proteins, such as catalytic subunit of DNA-PK (DNA-PKcs), Ku70, Ku86, Mre11, Xrcc4, and Nbs1 were also found to be associated with telomeric DNA (Um et al., 2004; Chin et al., 2005). These proteins play essential roles in repairing double-strand breaks (DSBs). Since age-dependent accumulation of DNA damage has been reported (Kirkwood and Finch, 2002), it is presumed that aging decreases the inherent capacity of DNA in somatic cells to remain stable and reduces the efficiency of the DNA repair systems. Indeed, an age-related decline in DNA double-strand break (DSB) repair was reported in unstimulated human lymphocytes (Mayer et al., 1991). Likewise, agedependent telomere shortening has been demonstrated in human CD4 T lymphocytes (Weng et al., 1995). Thus, the declining DSB repair capacity upon aging may underlie telomere shortening observed in human lymphocytes.

Based on these data, we reasoned that declining DSB repair capacity and increased telomere shortening in aged individuals may be associated with decreased expression of DSB repair proteins capable of telomere binding in the cell. To test this hypothesis, we obtained blood samples from various age groups of people living in Seoul, Korea and examined the level of DSB repair proteins in purified CD4+ T lymphocytes, a cell type which shows direct telomere shortening with age (Weng et al., 1995). Our data show that among the DSB repair proteins tested, only Ku70 and Mre11 are statistically significant age-dependent factors showing declining of cellular expression. Furthermore, Ku70 expression was significantly higher in the people living in the longevity community than those living in the control community. Collectively, our data show, for the first time, that Ku70 and Mre11 in human lymphocytes may represent new biomarkers for aging.

\section{Materials and Methods}

\section{Purification of human CD4 lymphocytes}

Human PBMCs (peripheral blood mononuclear cells) were obtained from 48 healthy volunteers of age between 20 and $80 \mathrm{yr}$ in Seoul area and from 80 healthy volunteers from Jeju area. All individuals provided written informed consent for blood donation and fully understood the experiment procedure. All the experimental protocols were reviewed and approved by institutional review boards.

CD4 positive $T$ cells were purified from peripheral blood lymphocytes (PBL). Briefly, $50 \mathrm{ml}$ of blood was collected from subjects and PBL was isolated from density gradient centrifugation using Ficoll-paque ${ }^{T M}$ PLUS (Amersham biosciences). CD4 positive T cells were purified using negative depletion kit (Miltenyi Biotec). The purity of CD4 T cells was examined using fluorescence activated cell sorter (FACs) anaIysis with CellQuest Pro Software (Becton Dickinson, NJ).

\section{Western blot analysis}

CD4 positive T cells were washed with phosphatebuffered saline (PBS) and lysed in RIPA lysis buffer (50 mM Tris- $\mathrm{HCl} \mathrm{pH} \mathrm{7.5,} \mathrm{1 \%} \mathrm{NP-40,} 150$ mM sodium 
chloride, $0.5 \%$ sodium deoxycholate and $0.1 \%$ SDS) with protease inhibitor cocktail (Roche). An equal amount $(20 \mathrm{ug})$ of total protein was loaded onto a $10-12 \%$ SDS-PAGE. After electrophoresis, proteins were transferred to a nitrocellulose membrane. Pro- teins were detected by monoclonal rabbit anti-human Ku70 or monoclonal rabbit anti-human Mre11 and horseradish peroxide (HRP)-conjugated goat anti-rabbit Ig using western blotting luminal reagent (Santa Cruz Biotechnology). Blots were stripped by
A
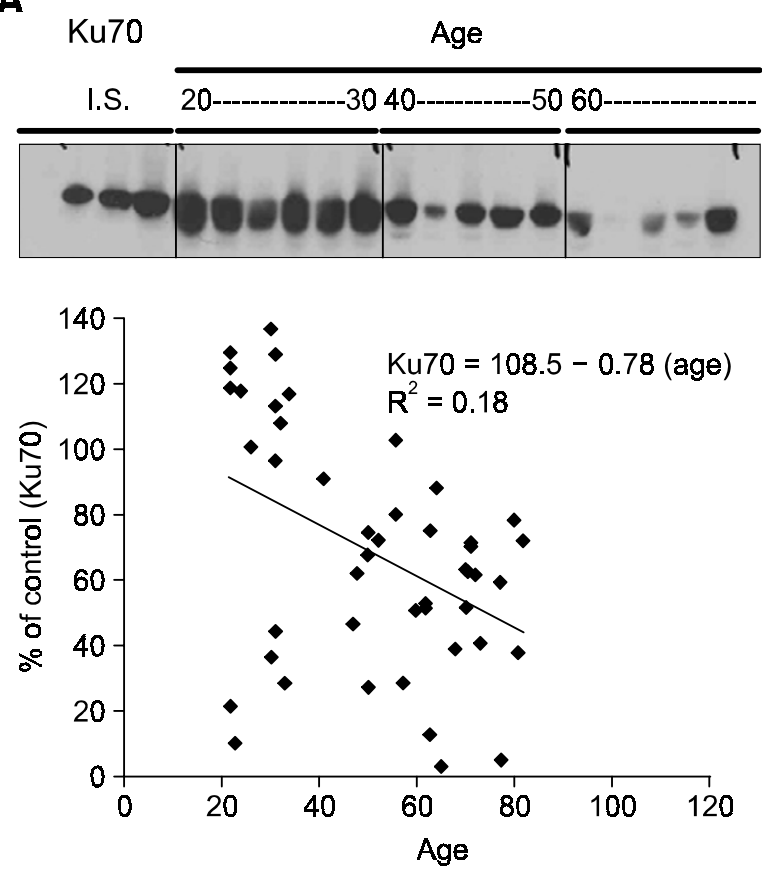

C Characteristics of study subjects in Seoul area

\begin{tabular}{cc}
\hline & Seoul area \\
\hline $\mathrm{N}$ & 48
\end{tabular}

$\begin{array}{ll}\text { Age }(\text { years })^{*} & 51.5 \pm 19.3 \\ { }^{*} \text { Mean } \pm \text { STD }\end{array}$

B

I.S. $\frac{\text { Age }}{20 \text { Mre11 }}$

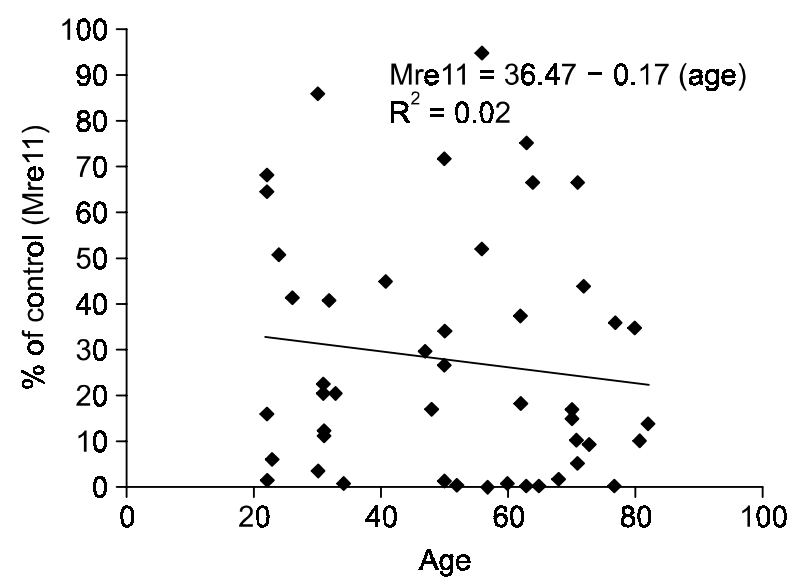

D Simple regression analysis of age on ku70 as well as on Mre11 in Seoul area

\begin{tabular}{|c|c|c|c|c|c|c|}
\hline \multirow[b]{2}{*}{ Variable* } & \multicolumn{3}{|c|}{ Ku70 } & \multicolumn{3}{|c|}{ Mre11 } \\
\hline & Coeff. & Std. Err. & $p$-value & Coeff. & Std. Err. & $p$-value \\
\hline Intercept & 108.50 & 13.48 & $<.0001$ & 36.47 & 10.92 & 0.0017 \\
\hline Age & -0.78 & 0.25 & 0.0027 & -0.17 & 0.20 & 0.3906 \\
\hline
\end{tabular}

Figure 1. Protein expression of Ku70 and Mre11 in CD4+ T cells obtained from 48 volunteers ranging from 20 to 80 yr old. Top panel; Total protein level of Ku70 (A) or Mre11 (B) isolated from human lymphocytes was determined by Western blots using rabbit monoclonal Abs against human Ku70 or human Mre11. Among the 48 samples tested, protein expression of 5-6 samples from each age groups (20-30, 40-50, 60 and over) was shown. I.S. stands for internal standards obtained from Ku-expressing cell line. 1, 4.5, and $20 \mu \mathrm{g}$ of lysates obtained from A549 human lung cancer cells were loaded onto the gel. Bottom Panel; Scatter plots of Ku70 (A) or Mre11 (B) protein level on age. Percentage of Ku70 or Mre11 expression in different aged individuals was obtained by dividing protein intensity of Ku70 or Mre11 by intensity of $20 \mu \mathrm{g}$ of internal standards shown above. (C) Characteristics of study subjects in Seoul area. (D) Result of the regression analysis of Ku70 on age, as well as that of Mre11 on age. 
stripping buffer (62.5 mM Tris- $\mathrm{HCl}$ (pH 6.8), 2\% SDS, and $100 \mathrm{mM}$ 2-mercaptoethanol). Then reprobed blots were detected by monoclonal rabbit anti-human $\beta$-actin. The density of bands was measured using Fujix-Bio-Imaging analyzer BAS2500 (Fuji Photo Film).

\section{Measurement of telomere length}

The mean telomeric restriction fragment (TRF) length was measured in human T-lymphocyte cells. Genomic DNA was extracted with DNeasy Tissue Kit (Qiagen, USA) and 5 ug of DNA was digested with Hinfl and Rsal (Boehringer Mannheim), and then electrophoresed through $1 \%$ agarose gels in $0.5 \times$ TBE buffer at $14^{\circ} \mathrm{C}$, using a CHEF MAPPER DRIII pulsed-field gel electrophoresis apparatus (Bio-Rad). Separation conditions were a ramped pulse speed of $1-6 \mathrm{~s}$ at $6 \mathrm{~V} / \mathrm{cm}$ for $16 \mathrm{~h}$. The gel was hydrolyzed for $5-10 \min$ in $0.25 \mathrm{M} \mathrm{HCl}$ and denatured for $1 \mathrm{~h}$ in $0.5 \mathrm{M} \mathrm{NaOH}$ and $1.5 \mathrm{M} \mathrm{NaCl}$. The gel was blotted and hybridized with a $\left[\gamma^{32} \mathrm{P}\right]$ labeled telomeric oligonucleotide probe $\left[\gamma^{32} \mathrm{P}-(\mathrm{CCCTAA})_{3}\right]$. After washing, the gel was autoradiographed on phosphor screen (Kodak) for $30 \mathrm{~min}$ and those lanes in which mean TRF was calculated were scanned using Personal Molecular Imager FX system (Bio-RAD). Mean TRF length was defined as: $\sum\left(O D_{i}\right) / \sum\left(O D_{i} / L_{i}\right)$ where $O D_{i}$ is the Personal Molecular Imager FX system output and $L_{i}$ is the length of the DNA at position $i$. The amount of telomeric DNA was calculated by integrating the volume of each smear in Quantity one software (Bio Rad).

\section{Statistical analysis}

Demographic characteristics for study subjects were represented by $n(\%)$ or mean \pm std as appropriately. Student's $t$-test was used to compare an age distribution between the longevity and control groups for subjects in Jeju area. A relation of age on Ku70, or that of Mre11, for the subjects in Seoul area was established by using a simple linear regression analysis. Effects of age and the longevity group on Ku70 were evaluated by using multiple regression analysis. Because there was no interaction effect between age and group on the expression of Ku70, main effect regression model was considered. Finally, a relationship between Ku70 and telomere length was examined by Pearson's correlation analysis. All statistical analyses were performed using SAS version 9.1 (SAS Institute Inc., Cary, NC). Reported $p$-values were two-sided, and $P<0.05$ was considered to be statistically significant.

\section{Results}

Declining of expression of two DSB proteins, Ku70 and Mre11, is associated with increased age

Since an age-related decline in DNA double-strand break (DSB) repair (Mayer et al., 1991) and agedependent telomere shortening (Weng et al., 1995) has been demonstrated in human lymphocytes, we examined if DSB repair proteins could show ageassociated changes in their expression. For this end, we collected blood from 100 different aged volunteers ranging from 20 to $80 \mathrm{yr}$ old living in Seoul, Korea. These individuals were all healthy with no visible signs of illness. CD4+ T lymphocytes were isolated from these individuals and the expression of telomere binding proteins, Ku70, Ku80, Sir2, TRF1, Mre11, were monitored by Western blot analysis using antibodies specific to each telomere binding proteins (Figure 1). Among these proteins tested, the protein level of Ku70 and Mre11 showed marked decline with respect to their age (Figure 1). As shown in Figrue 1, the protein level of Ku70 in individuals of 60 years old and over was only $50 \%$ of that in individuals of 20-30 $\mathrm{yr}$ old. A linear regression analysis of Ku70 (\% of control) on age shows that the rate of Ku70 declining is close to $-1(P=0.0027$, Figure 1D), indicating that expression of Ku70 is under the tight control upon aging. Similarly, expression of Mre11 was decreased with age, but the rate of decline was much slower than that of Ku70 (Figure 1B). All the other telomere binding proteins tested, including Ku80, Sir2, TRF1, did not show any age-dependent changes in their expression level (data not shown). We next determined if the change in Ku70 expression is associated with change in Mre11 expression. As shown in Figure 2, we found that the level of Ku70 expression was directly proportional to that of Mre11 expression. These data show that upon aging, CD4 T lymphocytes gradually lose their ability to keep high level of Ku70 and Mre11, which may result in partial impairment of DSB repair and telomere shortening.

\section{Slowed age-dependent declination of Ku70 in people living in the "longevity group"}

Since the cellular level of Ku70 decreases with age, we questioned if the maintenance of Ku70 expression may be partially responsible for keeping long life span in human. To directly test this idea, we searched the database from Korean National Statistical Office for the communities that are known to have the highest average lifespan in Korea. The 46 subjects aged between 40 to $80 \mathrm{yr}$ old in the longevity group were carefully selected from the families who have settled in the same community for 


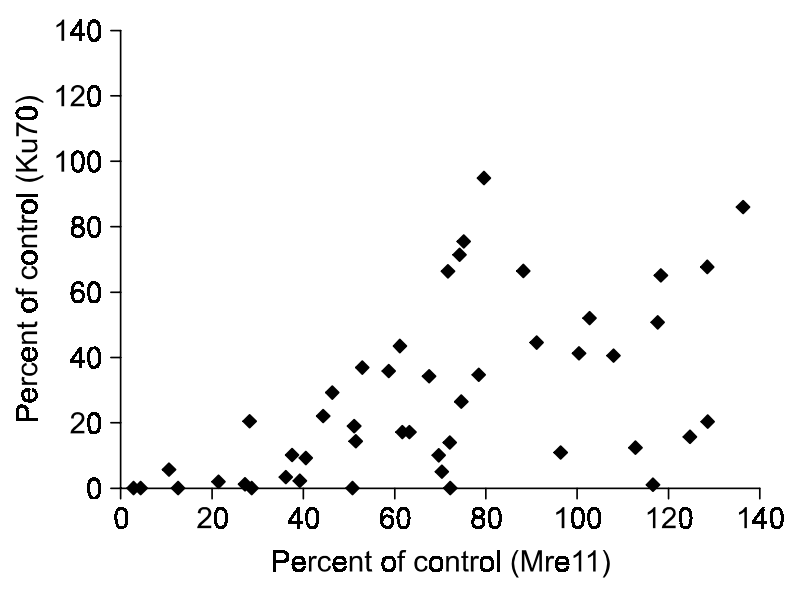

Figure 2. Correlation analysis of Ku70 and Mre11 expression in people aged between 20 and 80 yr. Scatter plots of Ku70 and Mre11 expression show significant linear relationship between Ku70 and Mre11 (Pearson's correlation coefficient, $r=0.5472, \quad P<$ .0001). Percentage of Ku70 or Mre11 expression in different aged individuals was obtained by dividing protein intensity of Ku70 or Mre11 by intensity of $20 \mu \mathrm{g}$ of internal standards obtained from Ku-expressing cell line, A549 human lung cancer cell lysates loaded onto the gel. more than $100 \mathrm{yr}$. This selection minimizes the influence from other environmental and stressful exposures to the individuals in the longevity community, so that the data obtained from the longevity group can truly represent the whole longevity group. Control subjects were chosen within 100 miles of the longevity community which show similar life patterns but with much shorter average lifespan. 34 subjects from the control group were evenly distributed between 40 and $80 \mathrm{yr}$ old. At the all ages tested, expression of Ku70 in the longevity group was significantly higher than that in the control group (Figure 3). Since there was no significant interaction effect between age and groups on Ku70 ( $P=$ $0.4684)$, the main effects regression model was used to find a relationship among age, groups and Ku70 (Figure 4). While the percentage of control in Ku70 declined significantly as age increased in both of the longevity and control groups $(P<.0001)$, it was significantly higher in the longevity group than the control group $(P=0.0322)$. These results imply that the intrinsic amount of Ku70 in individuals of these two groups was different from the birth. Therefore, it is tempting to speculate that people who have

A Ku70 Density plot

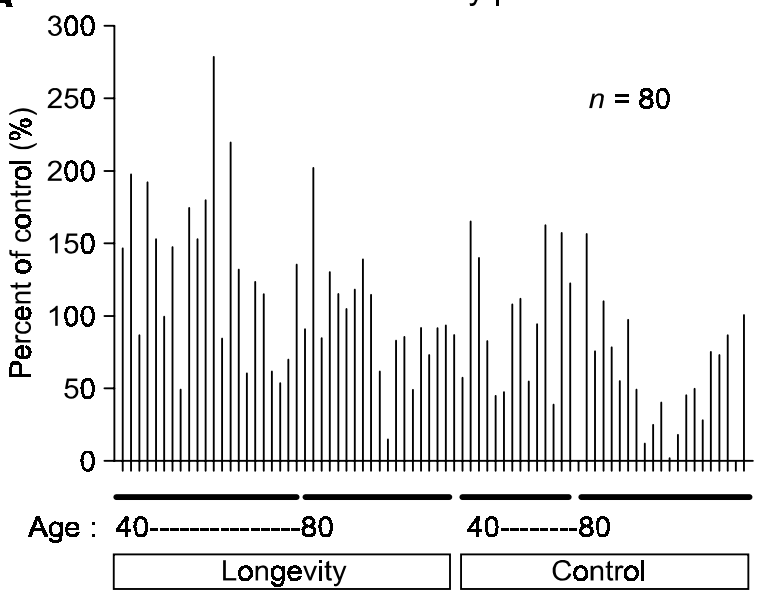

B Ku70 Western blot

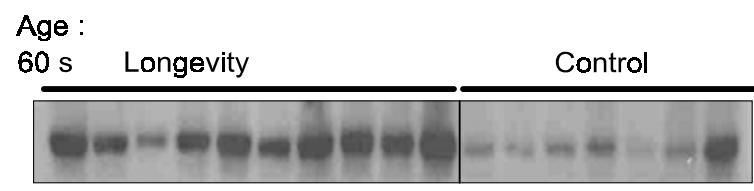

Age :

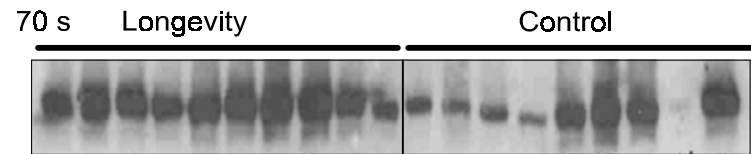

C

Characteristics of study subjects in jeju area

\begin{tabular}{cccc}
\hline & Jeju area, longevity group & Jeju area, control group & $P$ - value ${ }^{\dagger}$ \\
\hline $\mathrm{N}(\%)$ & $46(57.5)$ & $34(42.5)$ & \\
\hline Age (years) & $59.2 \pm 10.3$ & $63.8 \pm 8.9$ & 0.0401 \\
\hline
\end{tabular}

${ }^{*}$ Mean \pm STD ${ }^{\dagger}$ Student's $t$-test

Figure 3. Expression of Ku70 in CD4+ T cells obtained from the longevity group and the control group. (A), The protein level of Ku70 from 80 samples obtained from the longevity and the control group was expressed as a percentage of control (as described in Figure 1) and plotted as a bar graph against age. Characters or numbers on X coordinate are arbitrarily given subject designations. (B) Ku70 protein expression from the samples of $60 \mathrm{~s}$ and $70 \mathrm{~s}$ of old subjects was shown in anti-Ku70 immunoblots. (C), Characteristics of study subjects in Jeju area. There was no significant interaction effect between age and groups on Ku70 and the conclusion of our experiment $(P=0.4684)$. 
A $\mathrm{Ku} 70=223.8-2.07$ (age) (longevity group) $\mathrm{Ku} 70=202.26-2.07$ (age) (control group)

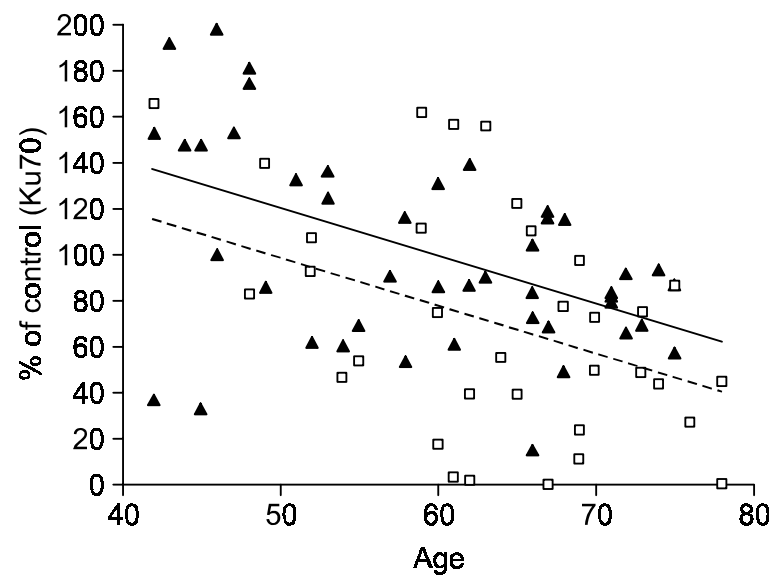

B Multiple regression analysis of age and longevity group on Ku70 in jeju area

\begin{tabular}{cccc}
\hline Variable $^{*}$ & Coeff. & Std. Err. & $P$ - value \\
\hline Intercept & 202.26 & 32.24 & $<.0001$ \\
\hline Age & -2.07 & 0.49 & $<.0001$ \\
\hline Longevity ${ }^{\dagger}$ & 21.54 & 9.88 & 0.0322 \\
\hline${ }^{*} \mathrm{R}^{2}=0.27,{ }^{\dagger}$ Reference $=$ control group &
\end{tabular}

Figure 4. Decreasing pattern of Ku70 with age for the control and the longevity group in Jeju area. (A) A scatter plot of Ku70 expression against age was shown for the longevity group and the control group, respectively. Percentage of Ku70 expression in individuals, obtained as described in Figure 1, was plotted against age. (B) A result for the multiple regression analysis with Ku70, as a dependent variable, and the age and group variables, as independent variables.

tendency to live long life span possess higher level of Ku70 protein, which is essential to maintain DSB repair activity.

\section{No direct correlation between the level of Ku70 expression and telomere length}

If the role of Ku70 is directly involved in the protection of telomeres, the length of telomere should be proportional to the cellular amount of Ku70. To test this, we measured mean telomere length from the nuclear extracts obtained from CD4+ T cells isolated from both longevity groups and the control groups and the individual telomere length was plotted against the level of Ku70. As shown in Figure 5, the telomere length measured in each individuals ranged anywhere between 5,000 to 8,000 bps. However, to our surprise, no significant correlation

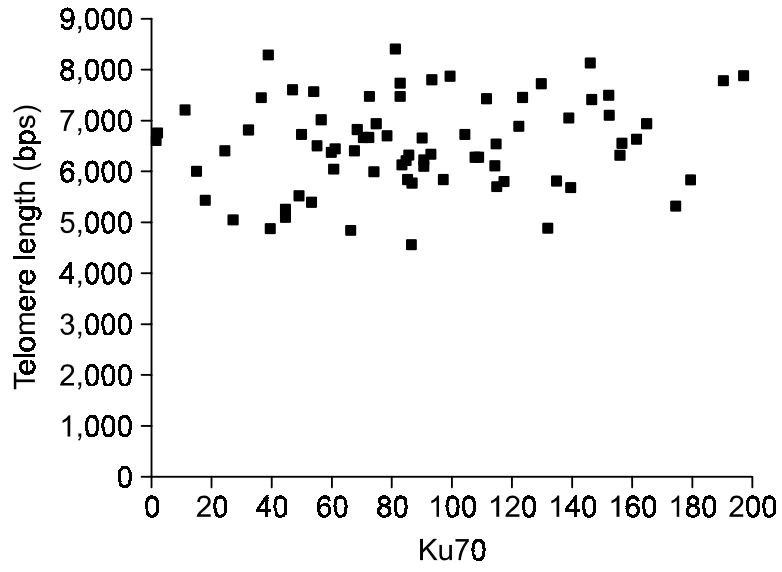

Figure 5. Scatter plot of Ku70 expression and the length of telomeres in Jeju area. Length of telomeres in CD4+ T cells was measured as described under Materials and Methods and plotted against expression of Ku70 (Pearson's correlation coefficient, $r=$ $0.1379, P=0.2350$ ). There was no statistically significant correlation between the level of Ku70 and the length of telomere.

was observed between the length of telomere and the level of Ku70 ( $r=0.138, P=0.2350)$. It suggests that Ku70 may not directly function to regulate the length of telomere, but rather affects other aspect of repair activity within the telomere region of the chromosome. Therefore, the maintenance of Ku70 levels may be critical for keeping longer life span by affecting mechanisms other than the lengthening of telomeres.

\section{Discussion}

The telomeres in human somatic cells undergo progressive shortening during cell division by replication-dependent loss of sequence at DNA termini. Maintenance and stabilization of telomeric DNA is assisted by binding of multiple DNA-binding proteins, including those involved in double strand break (DSB) repairs. Because functional telomeres are essential for continuous cellular proliferation and stable genetic inheritance, loss of chromosomal end capping has consequences in both aging and cancer (Harley et al., 1992; Artandi et al., 2000; DePinho and Wong, 2003). We discovered in this study that the total protein level of Ku70 declines in human lymphocytes with respect to the age (Figure 1A). Since an age-related decline in DNA double-strand break (DSB) repair (Mayer et al., 1991) and agedependent telomere shortening (Weng et al., 1995) was reported in human lymphocytes, decline of Ku70 expression seen in aged individuals may underlie the basis of telomere inconsistancy and cellular 
senescence. Furthermore, the level of Ku70 was significantly higher in the people in the community that has higher average lifespan than those living in the control community (Figures 3 and 4), again providing a correlation between the level of Ku70 and the lifespan. Together our data are in consistent with the idea that the cellular level of Ku70 in human lymphocytes reflects the biological age, and the loss of Ku70 may be critically leading to cellular senescence.

DSBs are potentially catastrophic lesions that if not repaired will lead to loss of genetic information and mutagenesis or cell death. In mammalian cells, two major pathways exist to repair DSBs; homologous recombination (HR) and nonhomologous end-joining (NHEJ; Liang et al., 1998). Several processes exist in which repair of a DSB is restricted to either NHEJ or HR. Ku70, existing as the Ku70 /Ku80 heterodimer complex, is a component of DNAdependent protein kinase (DNA-PK). Along with Ku80 and DNA-PKcs (DNA-PK catalytic subunit), Ku70 participates in DSB repair through NHEJ. Mutations in any of the component of DNA-PK result in endto-end fusions between chromosomes and deleterious terminal rearrangements (Bailey et al., 1999). Similarly, telomeric fusions and dysfunction was reported when Ku70 gene was deleted in a mouse (Bailey et al., 1999; d'Adda di Fagagna et al., 2001). Thus, loss of Ku70 in aging cells as seen in our study is likely to contribute to the telomere instability and telomere shortening process. Interestingly, the level of other proteins involved in DSB repairs, Ku80, Sir2, TRF1, did not show any age-dependent changes. These data indicate that not all telomerebinding proteins undergo tight regulation of its protein expression upon aging. However, Mre11, which is shown to exist as a complex with $\operatorname{Rad50}$ and Nbs1, undergoes age-dependent decline of its expression in human lymphocytes (Figure 1B). Mre11 complex was shown to influence recombinational DNA repair through promoting recombination between sister chromatids. Hypomorphic mutants of Mre11 have been shown to cause human genetic instability disorders (Stracker et al., 2004). Given the role of Ku70 and Mre11 in DSB repair and chromosome stability, age-dependent decline of Ku70 and Mre11 seen in our study may directly be associated with cell senescence and aging.

Aging is a process that changes healthy individuals into weak ones with decreased performance of most physiological systems and increased susceptibility of disease and death. The rate of aging and maximum lifespan varies among species, and therefore must be at least partly under genetic control (Miller et al., 2002; Hekimi and Guarente, 2003 ). It has been shown that up to $25 \%$ of the variation in human lifespan is inheritable (Herskind et al., 1996; Mitchell et al., 2001); the rest is due to environmental exposures, accidents and injuries, and chance. Very long life, to beyond age 90 years, appears to have an even stronger genetic basis (Perls et al., 2002), which explains why centenarians and near-centenarians tend to cluster in families. At the other extreme, the progeroid syndromes of accelerated aging and death at an early age have known genetic causes (Martin, 1985; Fossel, 2000). Although various regions of chromosomes 1, 2, 4, 7, 12 and 17 have been found to interact among themselves and with environmental factors to influence the median lifespan in 20 inbred strains (Gelman et al., 1988). Some of these chromosomes bear genes coding molecules with relevant functions in aging process. In this study, we are the first to show that the total protein expression of Ku70 decreases upon aging in human lymphocytes and the ability to retain Ku70, as shown in the longevity group, may partially contribute to the elongated lifespan observed in the longevity community. Decreased expression of Ku70 and Mre11 upon aging will not only impair the ability to repair DSBs, but also predisposes cells to the damage towards environmental factors including oxidative stress, ionizing radiations, toxic chemicals, etc.

Taken together, our data show, for the first time, that Ku70 and Mre11 may represent new biomarkers for aging and further suggest that maintenance of higher expression of Ku70 and Mre11 may be responsible for keeping longer life span observed in the longevity group.

\section{Acknowledgement}

We thank Dr. Jin-Won Hyun for providing lab space and FACs facility at Jeju University Medical School for purification of human CD4+ lymphocytes from the longevity and control groups. This work was supported by the Grant of the Ministry of Health and Welfare (A050331), Korean Research Foundation (KRF-2004-015-C00409) and the National Nuclear R\&D Program of the Ministry of Science and Technology of Korea (Grant BAERI).

\section{References}

Artandi SE, Chang S, Lee SL, Alson S, Gottlieb GJ, Chin L, DePinho RA. Telomere dysfunction promotes non-reciprocal translocations and epithelial cancers in mice. Nature 2000;406:641-5

Bailey SM, Meyne J, Chen DJ, Kurimasa A, Li GC, Lehnert BE, Goodwin EH. DNA double-strand break repair proteins are required to cap the ends of mammalian chromosomes. Proc Natl Acad Sci USA 1999;96:14899-904 
Bekaert S, Derradji H, Baatout S. Telomere biology in mammalian germ cells and during development. Dev Biol 2004; 274:15-30

Benard C, McCright B, Zhang Y, Felkai S, Lakowski B, Hekimi $\mathrm{S}$. The $\mathrm{C}$. Elegans maternal-effect gene clk-2 is essential for embryonic development, encodes a protein homologous to yeast Tel2p and affects telomere length. Development 2001;128:4045-55

Chin C, Bae JH, Kim MJ, Hwang JY, Kim SJ, Yoon MS, Lee MK, Kim DW, Chung BS, Kang CD, Kim SH. Radiosensitization by targeting radioresistance-related genes with protein kinase A inhibitor in radioresistant cancer cells. Exp Mol Med 2005;37:608-18.

d'Adda di Fagagna F, Hande MP, Tong WM, Roth D, Lansdorp $P M$, Wang ZQ, Jackson SP. Effects of DNA nonhomologous end-joining factors on telomere length and chromosomal stability in mammalian cells. Curr Biol 2001;11: 1192-6.

DePinho RA, Wong KK. The age of cancer: telomeres, checkpoints, and longevity. J Clin Invest 2003;111:S9-14

Fossel M. Human aging and progeria. J Pediatr Endocrinol Metab 2000;16:1477-81

Gelman R, Watson A, Bronson R, Yunis E. Murine chromosomal regions correlated with longevity. Genetics 1988;118: 693-704

Haendeler J, Hoffmann J, Diehl JF, Vasa M, Spyridopoulos I, Zeiher AM, Dimmeler S. Antioxidants inhibit nuclear export of telomerase reverse transcriptase and delay replicative senescence of endothelial cells. Circ Res 2004;94:768-75

Harley CB, Futcher AB, Greider CW. Telomeres shorten during ageing of human fibroblasts. Nature 1990;345:458-60

Harley CB. Telomere loss: mitotic clock or genetic time bomb? Mutat Res 1991;256:271-82

Harley CB, Vaziri H, Counter CM, Allsopp RC. The telomere hypothesis of cellular aging. Exp Gerontol 1992;27:375-82

Hastings R, Li NC, Lacy PS, Patel H, Herbert KE, Stanley AG, Williams B. Rapid telomere attrition in cardiac tissue of the ageing Wistar rat. Exp Gerontol 2004;39:855-7

Haussmann MF, Winkler DW, O'Reilly KM, Huntington CE, Nisbet IC, Vleck CM. Telomeres shorten more slowly in long-lived birds and mammals than in short-lived ones. Proc Biol Sci 2003;270:1387-92

Hekimi S, Guarente L. Genetics and the specificity of the aging process. Science 2003;299:1351-4

Herskind AM, McGue M, Holm NV, Sorensen TI, Harvald B, Vaupel JW. The heritability of human longevity: a population-based study of 2872 Danish twin pairs born 18701900. Hum Genet 1996;97:319-23

Jeon HY, Hyun SH, Lee GS, Kim HS, Kim S, Jeong YW, Kang SK, Lee BC, Han JY, Ahn C, Hwang WS. The analysis of telomere length and telomerase activity in cloned pigs and cows. Mol Reprod Dev 2005;71:315-20

Karlseder J, Smogorzewska A, de Lange T. Senescence in duced by altered telomere state, not telomere loss. Science 2002;295:2446-9

Kirkwood TB, Finch CE. Ageing: the old worm turns more slowly. Nature 2002;419:794-5

Liang F, Han M, Romanienko PJ, Jasin M. Homology-directed repair is a major double-strand break repair pathway in mam malian cells. Proc Natl Acad Sci USA 1998:95:5172-7

Martin GM. Genetics and aging; the Werner syndrome as a segmental progeroid syndrome. Adv Exp Med Biol 1985; 190:161-70

Mayer PJ, Lange CS, Bradley MO, Nichols WW. Gender differences in age-related decline in DNA double-strand break damage and repair in lymphocytes. Ann Hum Biol 1991;18:405-15

Miller RA, Harper JM, Galecki A, Burke DT. Big mice die young: early life body weight predicts longevity in genetically heterogeneous mice. Aging Cell 2002;1:22-9

Mitchell BD, Hsueh WC, King TM, Pollin TI, Sorkin J, Agarwala $R$, Schaffer AA, Shuldiner AR. Heritability of life span in the old order amish. Am J Med Genet 2001; 102:346-52

Nilsson P, Mehle C, Remes K, Roos G. Telomerase activity in vivo in human malignant hematopoietic cells. Oncogene 1994:9:3043-8

Perls T, Levenson R, Regan M, Puca A. What does it take to live to 100 ? Mech Ageing Dev 2002;123:231-42

Prowse KR, Greider CW. Developmental and tissue-specific regulation of mouse telomerase and telomere length. Proc Nat Acad Sci USA 1995;92:4818-22

Stracker TH, Theunissen JW, Morales M, Petrini JH. The Mre11 complex and the metabolism of chromosome breaks: the importance of communicating and holding things together. DNA Repair (Amst) 2004;3:845-54

Um JH, Kang CD, Bae JH, Shin GG, Kim DW, Kim DW, Chung BS, Kim SH. Association of DNA-dependent protein kinase with hypoxia inducible factor- 1 and its implication in resistance to anticancer drugs in hypoxic tumor cells. Exp Mol Med 2004;36:233-42.

van Steensel B, Smogorzewska A, de Lange T. TRF2 protects human telomeres from end-to-end fusions. Cell 1998; 92:401-13

Vaziri H, Dragowska W, Allsopp RC, Thomas RE, Harley CB, Lansdorp PM. Evidence for a mitotic clock in human hematopoietic stem cells: Loss of telomeric DNA with age. Proc Natl Acad Sci USA 1994;91:9857-60

Von Zglinicki T, Martin-Ruiz CM. Telomeres as biomarkers for ageing and age-related diseases. Curr Mol Med 2005; 5:197-203

Weng NP, Levine BL, June $\mathrm{CH}$, Hodes RJ. Human naive and memory $\mathrm{T}$ lymphocytes differ in telomeric length and replicative potential. Proc Natl Acad Sci USA 1995;92: 11091-4

Wright WE, Shay JW. Telomere-binding factors and general DNA repair. Nat Genet 2005;37:116-8 